\title{
Improving the Tea Withering Process Using Ethylene or UV-C
}

\author{
Emma R. Collings, M. Carmen Alamar, Maria Bogaerts Márquez, Sofia Kourmpetli, Zoltan Kevei, \\ Andrew J. Thompson, Fady Mohareb, and Leon A. Terry*
}

Cite This: J. Agric. Food Chem. 2021, 69, 13596-13607

Read Online

ACCESS | W W Metrics \& More | 国 Article Recommendations | st Supporting Information

ABSTRACT: Using a combination of biochemical, transcriptomic, and physiological analyses, we elucidated the mechanisms of physical and chemical withering of tea shoots subjected to UV-C and ethylene treatments. UV-C irradiation $\left(15 \mathrm{~kJ} \mathrm{~m}^{-2}\right)$ initiated oxidation of catechins into theaflavins, increasing theaflavin-3-monogallate and theaflavin digallate by 5- and 13.2-4.4-fold, respectively, at the end of withering. Concomitantly, a rapid change to brown/red, an increase in electrolyte leakage, and the upregulation of peroxidases (viz. Px2, $P x 4$, and $P x 6$ ) and polyphenol oxidases (PPO-1) occurred. Exogenous ethylene significantly increased the metabolic rate $(40 \%)$ and moisture loss $(30 \%)$ compared to control during simulated withering $\left(12 \mathrm{~h}\right.$ at $\left.25{ }^{\circ} \mathrm{C}\right)$ and upregulated transcripts associated with responses to dehydration and abiotic stress, such as those in the ethylene signaling pathway (viz. EIN4-like, EIN3-FBox1, and ERFs). Incorporating ethylene during withering could shorten the tea manufacturing process, while UV-C could enhance the accumulation of flavor-related compounds.

KEYWORDS: Camellia sinensis, transcriptome, de novo assembly, catechin, theaflavin, oxidation, polyphenols, peroxidase, abscisic acid, $E I N-4$

\section{INTRODUCTION}

Black tea accounts for $c a .75 \%$ of total world production. Its processing involves four stages: withering, fermentation (oxidation), cutting, and drying. ${ }^{1,2}$ Withering brings about both physical (water loss and leaf softening) and chemical changes (important for flavor and aroma development) and is regarded as a crucial stage in the tea manufacturing process. ${ }^{3}$ The methods used at each tea processing stage, in particular withering, have not been modified for many years and there is scope for improvement and/or optimization.

With the tea reference genome recently becoming available ${ }^{4}$ and several transcriptomic analyses published in recent years, ${ }^{5-7}$ the molecular mechanisms underpinning the responses of Camellia sinensis to a number of biotic and abiotic stresses, as well as its evolution and adaptation, ${ }^{8}$ are being elucidated. Thus, tea trancriptomic analyses have shed light on the gene regulatory responses during cold acclimation, ${ }^{9}$ gene regulatory networks for secondary metabolism, ${ }^{10}$ and the association between tea quality and stress response. ${ }^{11}$ Proteomic changes during tea withering have also recently been reported. ${ }^{12}$ However, there has been a lack of research focused on utilizing gene expression data to gain better mechanistic understanding of the black tea withering process.

Tea quality can be manipulated according to the "coarseness" of plucking. Shoots are typically harvested according to the "plucking standard" of "two leaves and a bud" (2LB), but coarse plucking (where three or four leaves are taken with the bud) is known to decrease quality because the larger and more mature leaves have lower catechin levels. ${ }^{13}$ During oxidation, catechins are converted to the flavor compounds theaflavins ( $\mathrm{TFs}$ ) by polyphenol oxidase (PPO), and then to thearubigins (TRs). The application of post- harvest treatments that enhance the oxidation of catechins into TFs and TRs may improve overall quality of the tea beverage.

Harvesting tea shoots triggers a cascade of senescencerelated processes. Leaf senescence is a complex process involving physiological, biochemical, and gene expression changes. ${ }^{14}$ Ethylene supplementation has been shown to enhance the senescence of a wide range of plant materials such as detached leaves, flower petals, and fresh cut lettuce. $^{15-20}$ Therefore, it was hypothesized that ethylene could increase the rate of tea shoot senescence making the withering process more rapid.

Changes in biochemical composition and enhanced senescence has also been observed with the application of UV radiation. However, effects of UV-A and UV-B on postharvest quality are less pronounced ${ }^{21}$ compared to those of UV-C application. Thus, low doses of UV-C have been studied on various fresh produce (as an abiotic elicitor) in an attempt to stimulate the production of defence-related compounds (hormesis), which can confer health promoting benefits to consumers (xenohormesis). ${ }^{22}$ Enhanced biochemical profiles have been reported for various fresh produce including table grapes, $^{23}$ fresh black pepper berries, ${ }^{24}$ and leafy vegetables. ${ }^{25}$ Consequently, low doses of UV-C could increase the amount of flavor compounds in a tea shoot. High doses of UV-C cause cell disruption (cell membrane damage) in a variety of fresh

Received: May 15, 2021

Revised: September 2, 2021

Accepted: October 4, 2021

Published: November 5, 2021

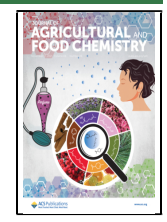


leaf produce including spinach and lettuce. ${ }^{26,27}$ Severe cellular damage inflicted by UV-C could be incorporated during tea processing to enhance the fermentation (catechin oxidation). However, work exploring the effects of UV-C on fresh tea leaves and subsequent changes during processing has not been reported to date.

In this work, the effect of ethylene supplementation and UV$\mathrm{C}$ exposure on the physiology and biochemical composition of fresh tea shoots during traditional withering conditions $\left(25^{\circ} \mathrm{C}\right.$ for 20 and $48 \mathrm{~h}$ ) is described and discussed in relation to tea quality. The genes regulating the observed biochemical changes are explored using transcriptomics to enable better mechanistic understanding of different treatment effects throughout the withering process.

\section{MATERIALS AND METHODS}

2.1. Plant Material. Two ethylene and two UV-C independent experiments were performed on tea shoots (two or three leaves and a bud [2LB or 3LB] shoot) harvested from Sri Lankan Clone 2 tea bushes (planted in May 1971) located in a small tea plantation based in Bedfordshire, UK. Experiments using ethylene (3LB) and UV-C (2LB) were performed over two years (years 1 and 2) for each treatment. Tea samples for the ethylene supplementation trial were harvested on 3rd October, 2013 and 30th July, 2014; whilst for UV-C experiments, tea shoots were harvested on 15th September, 2014 and 30th June, 2015, respectively. Different shoot standards (3LB vs 2LB) were used due to plant material availability at time of plucking. That said, the de novo transcriptome assembly and further transcriptome analysis was carried out using $2 \mathrm{LB}$ shoots from both experiments.

Immediately after plucking the tea shoots, samples were covered with a wet paper to prevent water loss (relative humidity between 85 and 95\%), and transported to Cranfield University, Bedfordshire, UK, within $2 \mathrm{~h}$ using an air-conditioned vehicle. Shoots remained at room temperature $\left(\right.$ ca. $\left.20^{\circ} \mathrm{C}\right)$ during transportation.

2.2. Experimental Design. 2.2.1. Ethylene Experiments. Upon arrival at Cranfield University, tea shoots for year 1 were randomly divided into six batches and placed into separate $(n=6)$ water-sealed air tight, $100 \mathrm{~L}$ polypropylene chambers. Samples were treated with continuous ethylene in air at $10 \mu \mathrm{L} \mathrm{L}^{-1}$, according to Alamar et al. $(2017),{ }^{28}$ or continuous air (control), at $25^{\circ} \mathrm{C}$ for $20 \mathrm{~h}$. Throughout storage, samples were removed from each box (at 0, 6, 12, and $20 \mathrm{~h}$ ) for physiological analysis (viz. respiration rate, objective color, and weight loss). For gene expression, samples were removed at 0, 2, 4, and $6 \mathrm{~h}$ and immediately snap frozen in liquid nitrogen and stored at $-80{ }^{\circ} \mathrm{C}$. Samples taken at 0 and $6 \mathrm{~h}$ were selected for transcriptome analysis.

Samples for year 2 were placed in 12 L Lock \& Lock polypropylene containers (L\&L Nordic OÜ, Estonia) $(315 \times 230 \times 230 \mathrm{~mm})$ and subsequently withered under continuous ethylene $\left(10 \mu \mathrm{L} \mathrm{L}^{-1}\right)$ or air, as previously described. At specific sampling points $(0,6,12$, and 20 h), six $3 \mathrm{LB}$ shoots were removed from three boxes per treatment for respiration rate and weight loss analysis.

2.2.2. UV-C Experiments. UV-C irradiation $(254 \mathrm{~nm})$ was applied at varying doses (0 [control], 1 [UV-C 1 and $15 \mathrm{~kJ} \mathrm{~m}^{-2}$ [UV-C $\left.\left.{ }_{15}\right]\right)$ using the same setup and protocol as described in Collings et al. (2018). ${ }^{24}$ Briefly, UV-C tubes (30 W, TUV-TL-D $254 \mathrm{~nm}$, Phillips, Surrey, UK) were mounted onto a custom-built rack, which was placed inside an enclosed fume hood for protection against exposure. The radiation delivered was measured using an optical radiometer (Multisense-100, Ultraviolet Products, Cambs., UK). The mean intensity (fluence rate) emitted by the UV-C setup was $8.3 \mathrm{~W} \mathrm{~m}^{3}$. During UV-C treatment, shoots were floated on cool water (ca. 11$13{ }^{\circ} \mathrm{C}$ ) to prevent overheating and excessive moisture loss. The abaxial (lower) surface of the leaf was placed in contact with the cool water whilst the dry adaxial (upper) surface was exposed to the UV-C light. During treatment application, control samples remained covered with aluminum foil for the whole treatment duration to prevent exposure to UV-C. In contrast, UV-C ${ }_{1}$ samples were covered with aluminum foil for the first $28 \mathrm{~min}$ and then uncovered for the last 2 min, whereas UV-C ${ }_{15}$ samples remained uncovered throughout the entire treatment duration. For years 1 and 2, UV-C doses of 0,1 , and $15 \mathrm{~kJ} \mathrm{~m}^{-2}$, and 0 and $15 \mathrm{~kJ} \mathrm{~m}^{-2}$ were applied, respectively. Leaf surface temperature did not exceed $20{ }^{\circ} \mathrm{C}$ during treatment application.

After UV-C treatment, shoots from both experiments were placed into $12 \mathrm{~L}$ Lock \& Lock polypropylene storage boxes as described above and stored under air conditions for up to 24 and $48 \mathrm{~h}$ for year 1 and 2, respectively. At each specific sampling point $(0,4,12,24$ and $48 \mathrm{~h}$ ), six shoots were removed from each box, where three shoots were used for respiration rate, ethylene production, and color assessment; two shoots for gene expression analysis; and one shoot for moisture content and conductivity (UV-C, year 2 only) measurements.

2.3. Physiological Measurements. 2.3.1. Respiration Rate. Real-time respiration rate measurements, were performed ex situ within $3 \mathrm{~L}$ jars using a sable respirometry system (model 1.3.8 Pro, Sable Systems International, Las Vegas, USA) as described in Collings et al. $(2018)^{24}$ with modifications. The sampled air from each jar was analyzed over a $2 \mathrm{~min}$ period, three times (cycles), to provide one mean reading at each outturn (sampling day). To prevent crosscontamination between samples, a 1 or 2 min baseline measurement was recorded which sampled air from the controlled temperature room at an identical flow rate. Initial $\mathrm{O}_{2}$ and $\mathrm{CO}_{2}$ levels were also used for the software calculations.

2.3.2. Weight Loss and Objective Color. Weight loss was recorded as absolute water content $(\%, \mathrm{w} / \mathrm{w})$ throughout the withering process. The initial percentage moisture content was assessed in triplicate on plucked tea shoots (six pseudo replicates [shoots] per replicate) immediately after arrival at Cranfield University. Shoots were dried at $70{ }^{\circ} \mathrm{C}$ for $24 \mathrm{~h}$ to obtain the dry weight.

Objective color (lightness $\left[L^{*}\right]$, chroma $\left[C^{*}\right]$, and hue angle $\left[h^{\circ}\right]$ ) was measured as described in Collings et al. (2019). ${ }^{3}$ The mean was calculated based on three readings measured from the largest leaf.

2.3.3. Water Potential, Moisture Content, and Conductivity. Water potential/water activity was determined using an AquaLab (Series 3, Version 1.7) dew point meter. A five point calibration curve was produced using known concentrations of glycerol to produce specific water activity readings (between 1 and 0.8 ). This allowed the $\%$ deviation between actual water activity value and meter reading to be calculated and sample readings were adjusted accordingly. Tea water activity was assessed using a disc-shaped sample cut from the middle section (including middle rib) of the largest leaf. Water activity values were converted to water potential using the formula described by Griffin (1994). ${ }^{29}$ The percentage moisture content was assessed on tea shoots, which were dried at $70{ }^{\circ} \mathrm{C}$ for $16 \mathrm{~h}$.

Conductivity was measured using a METTLER TOLEDO, SevenGo, with an InLab 738 probe $\left(0.01-1000 \mathrm{~ms} \mathrm{~cm}^{-1}\right.$ range) calibrated using a standard buffer solution (product no. 51-302-049) of known conductivity $\left(1413 \mu \mathrm{s} \mathrm{cm} \mathrm{cm}^{-1}\right)$. Measurements were performed using the smallest leaf after removal from the tea shoot. Leaves were placed directly in deionized water $(20 \mathrm{~mL})$ at $25^{\circ} \mathrm{C}$ and conductivity was measured after $1 \mathrm{~h}$. Total conductivity was determined by freezing the samples at $-80{ }^{\circ} \mathrm{C}$ followed by thawing and a further $2 \mathrm{~h}$ extraction at $25{ }^{\circ} \mathrm{C}$. Conductivity results were expressed as a percentage of total conductivity.

2.4. Biochemical Analysis. 2.4.1. Catechins, Methylxanthines, and TFs. Catechins and methylxanthines were extracted and quantified using the protocol described in Collings et al. (2018). ${ }^{24}$ TFs were quantified on the same extract according to ISO/DIS 18447. TF standards of known concentrations and undiluted samples were injected $(20 \mu \mathrm{L})$ into an Agilent ZORBAX Eclipse plus C18, $(4.6 \times 100 \mathrm{~mm}, 3.5 \mu \mathrm{m}$ particle size, part no. 959961-902) fitted with an OPTI-GUARD $1 \mathrm{~mm}$ guard column (part no. 51177-U). The mobile phase consisted of $2 \%$ acetic acid in acetonitrile (A) and $2 \%$ acetic acid $+20 \mathrm{mg} \mathrm{mL}^{-1}$ EDTA in water (B). Separation was achieved using an isocratic gradient of $20 \%$ solvent $\mathrm{A}$ and $80 \%$ solvent $\mathrm{B}$, for $20 \mathrm{~min}$ at a flow rate of $1.8 \mathrm{~mL} \mathrm{~min}^{-1}$ and a column temperature of $30^{\circ} \mathrm{C}$. Compounds were detected using a photodiode 
array detector (model G1315D) set to a wavelength of $274 \mathrm{~nm}$. Peak identification and quantification was achieved by comparison with known standards [TF, theaflavin-3-gallate, theaflavin-3'-gallate, and theaflavin digallate (TD)] purchased from Sigma (Dorset, UK).

2.5. RNA Extraction. Control and treated samples collected for RNAseq analysis at each sampling point (UV-C experiment: 0,4 , and $12 \mathrm{~h}$; ethylene experiment: 0 and $6 \mathrm{~h}$ ) were snap-frozen in liquid nitrogen and immediately stored at $-80{ }^{\circ} \mathrm{C}$ prior to analysis. Frozen shoots (2LB for both ethylene and UV-C experiments) were ground into a fine powder using a Retsch CryoMill (Dusseldorf, Germany) and RNA extracted from $c a .100 \mathrm{mg}$ FW powder using an RNeasy plant mini kit (Qiagen, Germany) and RNase-free DNase digestion (to eliminate genomic DNA) following manufacturer's instructions. The RNA quality was assessed using an Experion RNA chip (Bio-Rad, Hertfordshire, UK) to determine $28 \mathrm{~S} / 18 \mathrm{~S}$ ribosomal RNA ratio. High quality RNA (RQI > 8.5) from three biological replicates for each sample was used for transcriptome sequencing using Illumina HiSeq 2000 platform. Library preparation and sequencing was performed at Earlham Institute (Norwich, UK).

2.6. Transcriptomic Data Analysis. A total of 27 paired-end libraries were sequenced over five lanes generating more than 2049 million of $100 \mathrm{bp}$ reads (average of $76 \mathrm{M}$ reads per replicate). Quality assessment of the raw reads was performed using FastQC (version $0.11 .2) .{ }^{30}$ Reads were then filtered using Trimmomatic v.0.36 to remove sequencing adapters and trailing low-quality bases. A sample average of $92.5 \%$ of reads remained following filtration, resulting in a total of $1837 \mathrm{M}$ reads for de novo transcriptome assembly. Trimmed sequence read files were assembled using a Trinity suite v.2.1.1 ${ }^{31}$ using two k-mer sizes of 25 and 32 with normalized reads (maximum coverage $=50) .{ }^{32}$ The 25 -mer and 32 -mer assemblies were merged into a fast formatted file, and duplicated contigs between the two assemblies were removed using the CD-HITS-EST with an e-value threshold of $0.955^{33}$ An incremental filtration by transcripts per million (TPM) values (1,3, or 5) was performed to remove contamination, assembly artefacts, and transcripts with very low abundance, resulting in assemblies A4, A5, and A6, respectively (see Supporting Information Table S1). Sample read files were aligned to the merged assembly using $\operatorname{STAR}^{34}$ and transcripts with low abundance were removed using a threshold of 5 TPM, a value which provided the optimal number of contigs.

The assembled transcriptome was annotated using Blast2GO v4.1.9, ${ }^{35}$ by performing a BLASTx search against NR, KEGG, and TAIR10 databases using an $e$-value $\leq 10^{-3}$. Non-redundant transcripts were then functionally annonated using gene onthology (GO) terms based on the best BLASTx hit from the NR database. Transcripts were also annotated with protein functions using InterProScan $5 .{ }^{36}$

The de novo abundance estimation was performed using RSEM version 1.2.18. ${ }^{37}$ Filtered sample reads were mapped against the assembled transcriptome using STAR aligner. Transcripts supported by less than 20 reads or 5 TPM were excluded. Differential expression analysis was performed using $\mathrm{R}$ v3.4.0 with Bioconductor Limma v3.34.6. Raw counts were normalized using the "voom" algorithm with quality weights. ${ }^{38}$

In addition to the de novo transcriptome assembly, $a b$ inito transcriptomics was also performed using the publicly available tea tree genome ${ }^{39}$ and the Tuxedo suite. The tea genome was obtained from the Tea Tree Genome Database in FASTA format, ${ }^{40}$ an index reference sequence was created using Bowtie $2^{41}$ and filtered reads were aligned to the reference genome using Tophat. ${ }^{42}$ Next, Cufflinks, Cuffmerge, and Cuffdiff ${ }^{43}$ were used sequentially in order to generate a differential expression matrix. The output from the de novo and $a b$ initio transcriptomics were then merged into a single downstream analysis. First, a BLAST database was created using the merged annotation from the Cuffmerge step using a custom bash script, and the de novo transcripts from the final transcriptome assembly (A6) were blasted against this database using an e-value threshold of $10^{-3}$. Because our transcriptome assembly was generated at a much higher read depth (2049 vs 11Gb RNA-Seq reads) and annotated against multiple resources, it was eventually used for the remaining part of the downstream analysis. However, a lookup table linking A6's transcript
IDs to the tea tree genome ID is provided as Supporting Information data.

Unsupervised exploratory principal component analysis (PCA) was performed for the resulting data set. Once a linear model was fit using the design matrix, a series of contrast matrices were created with the aim to find the differentially expressed (DE) transcripts between ethylene-treated samples and control samples, or between different sampling points throughout the storage period. DE transcripts were considered significant at $P \leq 0.05$ and $\log$ fold change $(\log 2) \geq \pm 1.0$.

2.7. Statistical Analysis. For UV-C data, statistical analyses were carried out using Statistica for Windows version 10, 64-bit (Statsoft Tulsa, OK 74104, USA). Analysis of variance (ANOVA) was used to identify any significant differences $(p<0.05)$ between treatments followed by post-hoc analysis using Tukey's HSD test.

For ethylene, data were analyzed using Genstat software version 15. Analysis of variance (ANOVA) was used to identify the main effects of treatment, time course, and tissue to a probability of $p<0.05$, unless otherwise stated. Normality and homoscedasticity tests were performed on every variable to confirm the normality of the residues and the equality of the variance. For those few variables that did not meet these assumptions, a logarithm transformation on the raw data was carried out prior to ANOVA.

For gene expression analysis, a multivariate analysis (viz. PCA) was performed using Limma package in $\mathrm{R}$, prior to downstream analysis to assess similarities between time points and treatments and identify any potential outliers between sample point replicates. Heat maps were generated using Morpheus online software [accessed Dec 2019 from https://software.broadinstitute.org/morpheus/].

\section{RESULTS}

3.1. Tea Withering De Novo Transcriptome Assembly. 3.1.1. Tea RNA Exploratory Analysis. Unsupervised exploratory analysis with PCA showed clear clustering of all RNA sample replicates belonging to the same time point (Supporting Information Figure S1). This was further confirmed by the heat map hierarchical clustering; it showed close relationships between biological replicates, suggesting low biological variation within the plant material used for this study (Supporting Information Figure S1B). A good separation between withered samples and samples analyzed before withering was also found, regardless of the treatment (e.g., ethylene and UV-C), suggesting a similar expression pattern within the clusters (Supporting Information Figure S1). During withering, the samples were clustered according to the treatment and differentiated from their respective controls; however, there was no clear separation based on the withering time (Supporting Information Figure S1).

3.1.2. De Novo Transcriptome Assembly and Functional Annotation. RNA-seq data were obtained from 27 samples, including ethylene-treated $(n=3)$, UV-C treated $(n=6)$ and control for each of the two treatments $(n=9$ and 9 for the ethylene and UV-C, respectively). For the UV-C trial, each sample consisted of two $2 \mathrm{LB}$ tea shoots, whilst six shoots per sample were taken for the ethylene trial. The final transcriptome assembly (A6) achieved N50 of 1993, a total number of 57,232 transcripts, and 27,128 contigs with a length $\geq 1 \mathrm{kbp}$ (Supporting Information Table S1). 79\% of the transcripts were annotated based on BLAST hits against known transcripts. 35,103 transcripts were annotated with BLAST description and GO annotation, 5606 transcripts annotated with corresponding enzyme codes, and 4602 transcripts with only BLAST description (Supporting Information Figure S2).

3.1.3. Gene Ontology Analysis. Gene ontology (GO) classification of the de novo tea withering transcriptome 

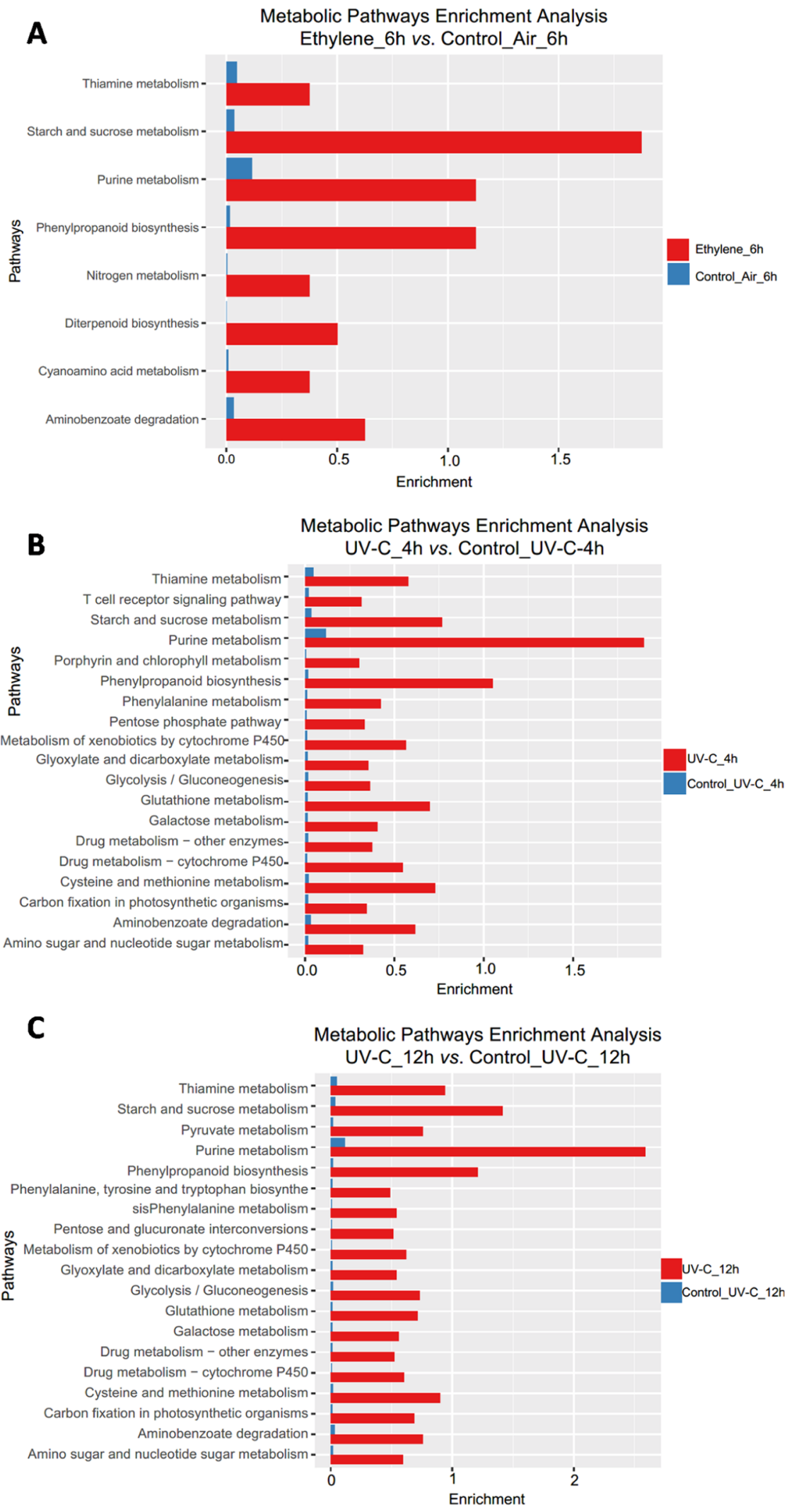

Figure 1. Metabolic pathway enrichment analysis for ethylene treatment (ethylene $6 \mathrm{~h}$ ) vs. control (control air $6 \mathrm{~h}$ ) fresh $\mathrm{C}$. sinensis $2 \mathrm{LB}$ shoots $\mathrm{C}$. sinenesis 2. (A); UV-C treatment at $4 \mathrm{~h}$ (UV-C_4h) vs control at $4 \mathrm{~h}$ (control_UV-C_4h) (B); and UV-C treatment at $12 \mathrm{~h}$ (UV-C_12h) vs control at $12 \mathrm{~h}$ (UV-C_4h) (C). $x$ axes indicate fold changes. Individual pathway ratios are available as Supporting Information Table S3.

(Supporting Information Figure S3) showed that a total of 40,709 unigenes were classified into biological process and molecular function categories. Genes involved in oxidation- reduction, metabolic processes, and regulation of transcription were highly represented in the biological process category (Supporting Information Figure S3A). Functional classification 
A.

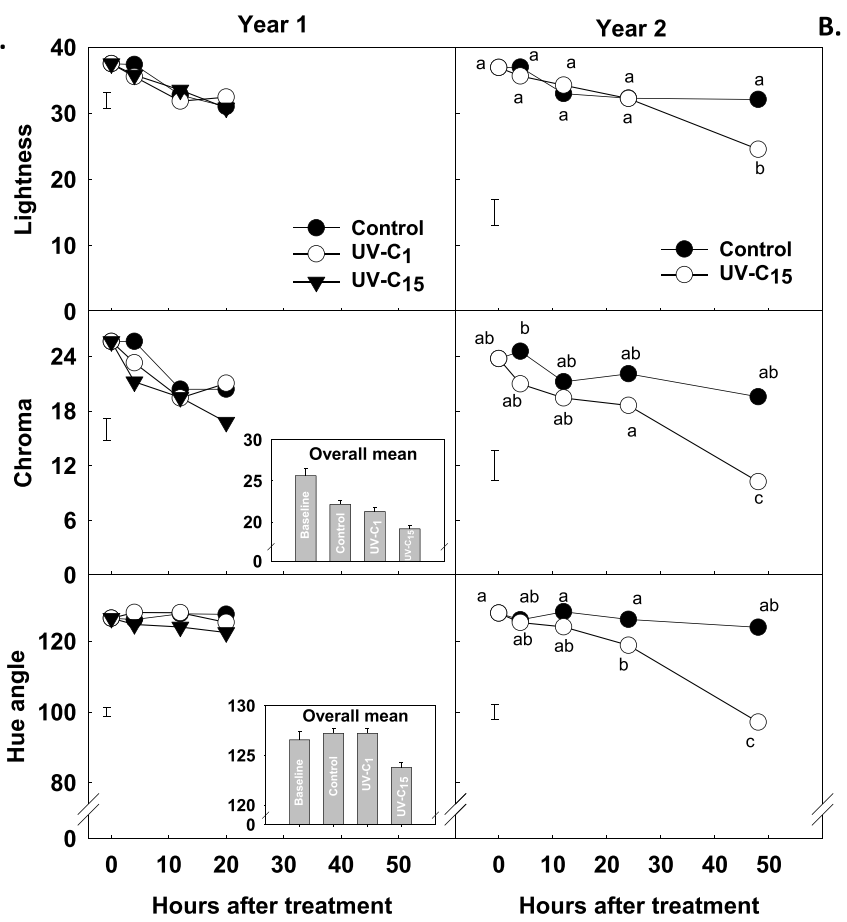

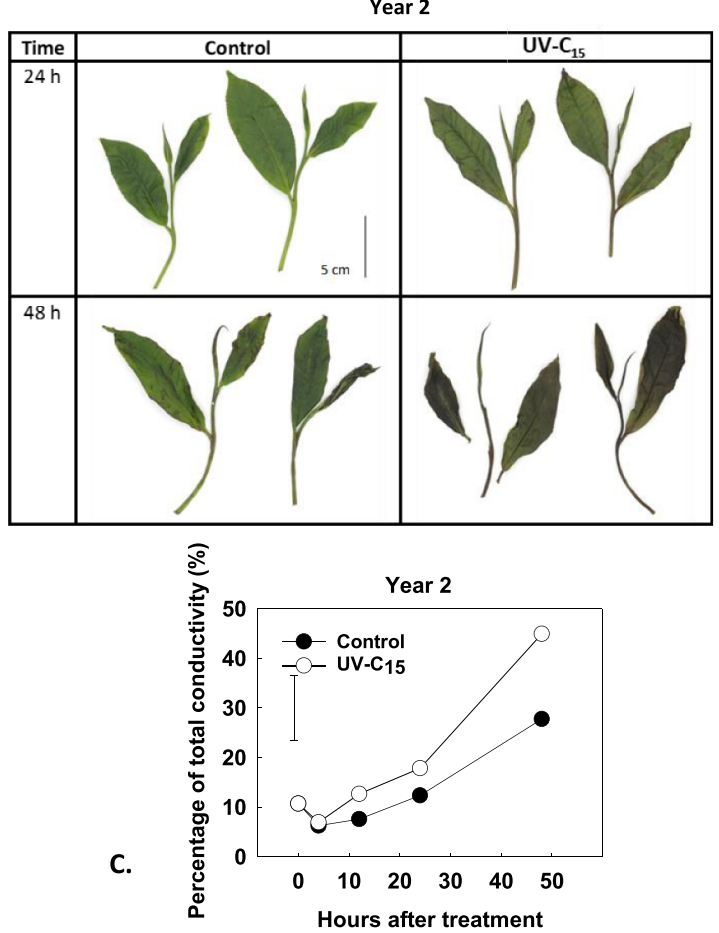

Figure 2. Leaf color and cell electroconductivity in UV-C treated C. sinensis $2 \mathrm{LB}$ shoots. Effect of UV-C dose treatments (UV-C ${ }_{1}\left[1 \mathrm{~kJ} \mathrm{~m}^{-2}\right]$; UV$\left.\mathrm{C}_{15}\left[15 \mathrm{~kJ} \mathrm{~m}^{-2}\right]\right)$ in comparison to control on lightness, chroma, and hue angle [A]; visual appearance [B]; and conductivity [C], in fresh tea leaves of clone 2 during $20 \mathrm{~h}$ (year 1) or $48 \mathrm{~h}$ (year 2) of storage at $25^{\circ} \mathrm{C}$; $n=3$. Different lower case letters represent significant differences between data points. Least significant difference $\left(\mathrm{LSD}_{0.05}\right)$ bars for the interaction treatment $\times$ hours after treatment are shown.

of the annotated unigenes in molecular function category (Supporting Information Figure S3B) revealed that hydrolase activity and DNA binding were among the highly represented groups. Furthermore, a total of 5606 transcripts were annotated with Enzyme Commission (EC) classification codes. According to this classification, a high proportion of assembled transcripts belonged to the transferases and oxidoreductases classes, representing 35 and $17 \%$, respectively (Supporting Information Figure S3C).

3.1.4. Differential Expression and Pathway Enrichment Analyses. All possible contrasts of control versus treatment were assessed and the number of transcripts that were up- or downregulated are shown in Supporting Information Table S2. Interestingly, the ethylene treatment appeared to have a limited effect on the gene expression profile of the withered tea leaves, with only 169 transcripts being DE (135 upregulated, 34 downregulated) after $6 \mathrm{~h}$ of ethylene treatment compared to the air-withered control. In contrast, UV-C treated tea leaves showed 17,889 transcripts $\mathrm{DE}$ compared to the respective control (Supporting Information Table S2). DE transcripts from UV-C treated samples were mapped to specific pathways related to ion channels, calcium transport, phenylalanine ammonia lyase (PAL), oxidation, and flavonoid synthesis that may be involved in the catechin and TF changes following UV-C treatment. Furthermore, ethylene perception, signal transduction, and catabolism pathways were explored for samples exposed to ethylene during simulated withering.

Metabolic pathway enrichment analysis showed eight overrepresented pathways in ethylene-treated tea leaves; including starch and sucrose metabolism, purine metabolism, phenylpropanoid biosynthesis, and aminobenzoate degradation (Figure 1A). In contrast, the UV-C treatment appears to affect a wider range of metabolic pathways with the effect being higher after $12 \mathrm{~h}$ of treatment compared to 4 (Figure 1B,C).

3.2. UV-C Caused Browning of Leaves and Cellular Damage after $48 \mathrm{~h}$. For UV-C treated shoots, storage at 25 ${ }^{\circ} \mathrm{C}$ for $20 \mathrm{~h}$ (year 1 ), resulted in samples treated with $15 \mathrm{~kJ}$ $\mathrm{m}^{-2} \mathrm{UV}-\mathrm{C}\left(\mathrm{UV}-\mathrm{C}_{15}\right)$ having an overall significantly lower $\mathrm{C}^{*}$ and $h^{\circ}$ compared to control and samples exposed to $1 \mathrm{~kJ} \mathrm{~m}^{-2}$ $\left(\mathrm{UV}-\mathrm{C}_{1}\right.$ ) (Figure $\left.2 \mathrm{~A}\right)$. An additional $28 \mathrm{~h}$ of storage during year 2 showed greater differences in color between treatments (Figure $2 \mathrm{~A}$ ) with $L^{*}, C^{*}$ and $h^{\circ}$ significantly lower in UV-C $\mathrm{C}_{15}$ compared to control, indicative of a darker brown/red color (Figure 2B). Electroconductivity (as \% of total conductivity), is a measure of cell disruption and was found to be overall significantly higher in UV-C treated shoots $(20.6 \%)$ compared to control (13.5\%) during $48 \mathrm{~h}$ storage at $20{ }^{\circ} \mathrm{C}$ and $90 \% \mathrm{RH}$ (year 2) (Figure 2C).

3.3. Biochemical Changes in Tea Shoots Following UV-C Treatment. Changes in biochemical content were assessed in both years. Whole shoots were analyzed in year 1 , while bud and large leaf were separated for year 2 (Supporting Information Figure S4). Gallic acid (GA) and catechin content was overall significantly higher in the bud, with the exception of gallocatechin (GC), epicatechin (EC), and (+)-catechin. Furthermore, both spatial regions showed reciprocal decreasing and increasing trends during simulated withering for catechins and GA, respectively (Supporting Information Figure S4).

UV-C treatment applied to tea shoots in year 1 and withered for $20 \mathrm{~h}$, showed no significant differences in catechin content between treatments, except for $(+)$-catechin where UV-C $\mathrm{C}_{15}$ treated shoots had overall higher concentrations (ca. 2.5-fold)

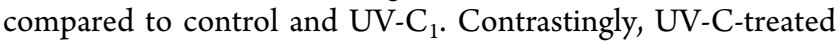
shoots withered for $48 \mathrm{~h}$ (year 2) experienced significantly 
lower catechin concentrations compared to control for epigallocatechin gallate (EGCG), epicatechin gallate (ECG), and GC (Figure 3 and Supporting Information Figure S4). GA
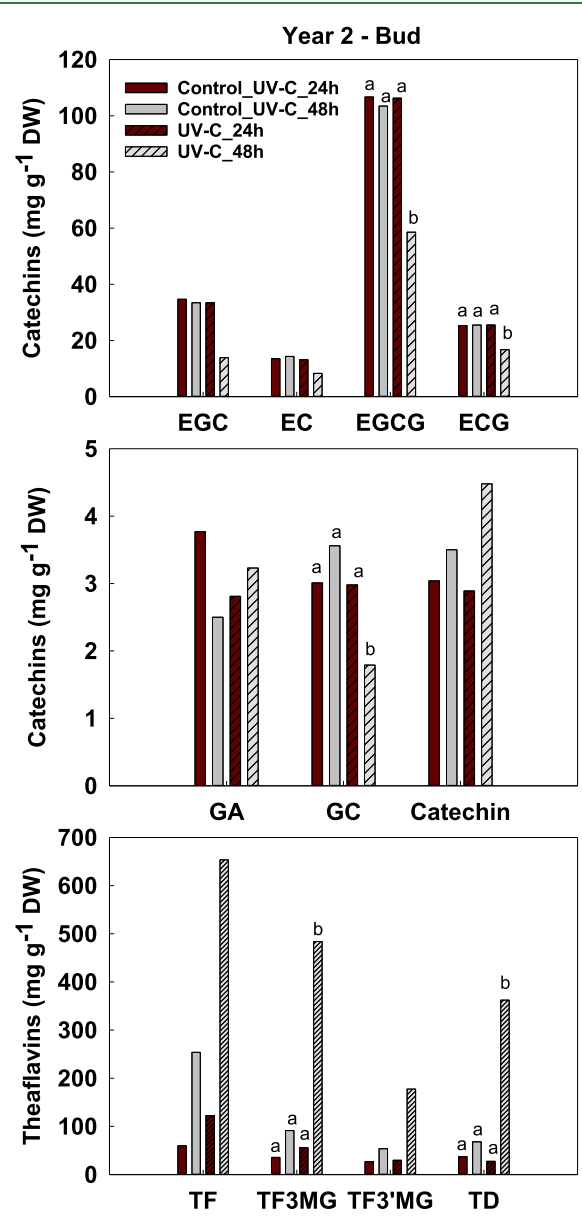

Figure 3. Catechin and theobromine concentration in C. sinensis buds. Effect of high UV-C dose treatment (UV-C $\left[15 \mathrm{~kJ} \mathrm{~m}^{-2}\right]$ ) in comparison to control (control_UV-C) on GA, catechin [EGCepigallocatechin; EC-epicatechin; EGCG-epigallocatechin gallate; ECG-epicatechin gallate; GC-gallocatechin; (+)-catechin], and theaflavin [TF-theaflavin; TF3MG-theaflavin 3-monogallate; TF3'MG - theaflavin-3'monogallate; TD-theaflavin digallate] content in fresh tea leaves of clone 2 (year 2 ; bud) at the last two sampling points $\left(24\right.$ and $48 \mathrm{~h}$ ) following storage at $25{ }^{\circ} \mathrm{C}$. Different lower case letters represent significant differences between data points; $n=3$.

levels also increased in all samples during the withering process but with no differences between treatments. An opposite pattern to that observed for catechins occurred for TFs where values increased during withering; however, significant differences between treatments only occurred after $48 \mathrm{~h}$ of simulated withering (Figure 3). At $48 \mathrm{~h}$ in both experiments, $\mathrm{UV} \mathrm{C}_{15}$ shoots had significantly higher theaflavin-3-monogallate (TF3MG) (5.8- and 6.6-fold) and TD (13.2- and 4.4fold) compared to controls in year 1 (whole shoot-data not shown) and year 2 (Figure 3), respectively. It should be noted that there was a difference of $c a .28 \mathrm{~h}$ between the last sampling point from each experiment (i.e. year 1 finished at 20 $\mathrm{h}$, and year 2 finished after $48 \mathrm{~h}$ ). UV-C had no effect on methylxanthine (caffeine and theobromine) content (data not shown); these are related alkaloids found in tea with important and characteristic stimulating properties.
3.4. Differential Gene Expression Associated with UV-C Exposure Prior to Simulated Withering. 3.4.1. Overall Treatment Effects Control Versus UV-C. The majority of transcripts upregulated in tea shoots exposed to UV-C followed by simulated withering were annotated as peroxidases (PO) from the subfamily of secreted class III peroxidases (Figure 4A), which are responsible for catalyzing the oxidation of substances by hydrogen peroxide. That said, a peroxidase 4like splice variant: Px4-like-2, was overall downregulated relative to the control. Fifteen transcripts attributed to an ion channel function were DE in UV-C treated shoots (Figure 4C). Most transcripts were upregulated by UV-C treatment; however, some ion channel transcripts showed a down regulation compared to control including CNG4, MS10-like1 , and potassium transporter POT7. Ten genes annotated as $P A L$, an enzyme responsible for catalyzing the first key step in the phenylpropanoid pathway, were all upregulated in response to UV-C treatment (Figure 4B). Seventeen transcripts related to the flavonoid biosynthesis pathway were DE between UV-C and control samples (Figure 4D); of these, fourteen were upregulated in UV-C treated samples.

3.4.2. Time Versus Treatment Interactions. As simulated withering time progressed, the expression of 36 peroxidase transcripts displayed an increasing pattern with most transcripts showing greater upregulation in UV-C samples after 12 $\mathrm{h}$ relative to control. In addition, two splice variants of polyphenol oxidase $(P P O)$ were annotated and upregulated in UV-C samples relative to control after $12 \mathrm{~h}$ wither, while peroxidase $6(P x 6)$ was upregulated by $c a .5-6 \log \mathrm{FC}$ at both withering time points. Expression of transcripts attributed to ion channel function (and electrolyte transport) were also found to increase with withering time in UV-C treated samples relative to the control. This comprised of four ion channel transcripts including splice variants: cyclic nucleotide gated channel CNG1-2, mechanosensitive (MS) ion channel such as MS10-like-2 and MS10-like-3 which showed significantly higher fold increases after $12 \mathrm{~h}$ simulated withering (4.9-7.7-fold log FC) compared to $4 \mathrm{~h}$ withering (1.9-3.0-fold log FC).

From the $10 \mathrm{PAL}$ transcripts identified (Figure 4), those annotated as PAL-4-1 and PAL-5 had the highest expression (3.2-3.5-fold log FC) in UV-C treated samples relative to control; however, PAL-4-1 showed greater expression after 12 $\mathrm{h}$ withering (3.5-fold $\log$ FC) compared to $4 \mathrm{~h}$ (2.5-fold $\log$ FC). Toward the latter stages of the phenylpropanoid pathway, the flavonoid transcript with the highest fold change (log FC of ca. 5) was annotated as flavonoid 3-monooxygenase-like F3'MO-like-1. This was closely followed by leucoanthocyanidin dioxygenase LDOX-1, LDOX-like-1-1, LDOX-like-1-3 in UV-C treated samples after $12 \mathrm{~h}$ wither, while malonyl flavonoid malonyltransferase MFMT5-1 and MFMT5-2-2 showed ca. 4fold $\log$ FC in UV-C samples after $4 \mathrm{~h}$.

3.5. Enhanced Leaf Senescence Using Exogenous Ethylene Application Shortens the Withering Process. Color measurements performed on the large leaf (third leaf) revealed a steady change from an intense green color to dull green/slightly yellow during withering, which was not affected by ethylene. This was objectively monitored by a gradual decrease in $h^{\circ}$ toward the end of the withering process where values dropped from 128.8 to 127.4 , respectively (year 1 ). At the end of the withering process, ethylene-treated samples had significantly lower (ca. 0.7-fold) absolute water content and 1.4-fold higher respiratory rate compared to control (air 
A
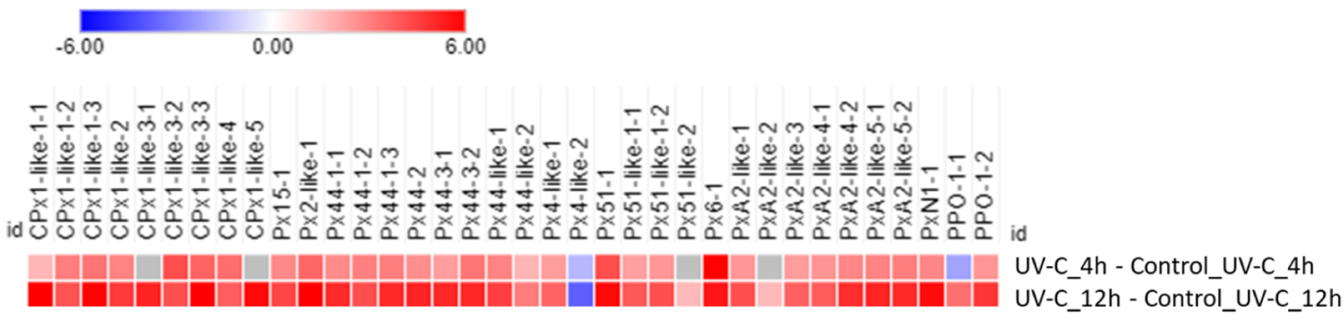

B

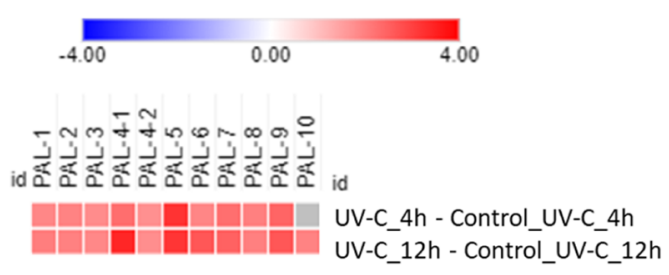

C

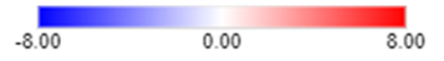

D

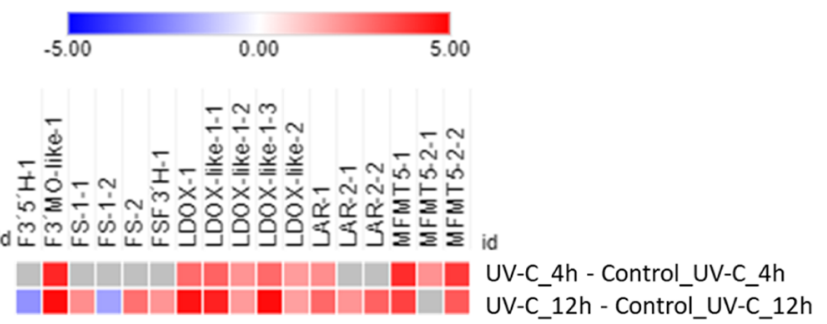

Figure 4. DE transcripts of $C$. sinensis $2 \mathrm{LB}$ shoots during simulated UV-C withering at 4 and $12 \mathrm{~h}$ (UV-C_nh) compared to their respective control (control_UV-C $n \mathrm{~h}$ ). Heat maps showing significant fold changes in [A] oxidation, [B] PAL, [C] ion channel, and [D] flavonoids transcripts between UV-C treated and control samples. $[C P x]$ cationic peroxidase; $[P x]$ peroxidase; $[P P O]$ polyphenol oxidase; $[P A L]$ phenylalanine ammonia lyase; $[C N G C]$ cyclic nucleotide gated channel; $[M S]$ mechanosensitive ion channel; $[P O T]$ potassium transporter; $\left[F 3^{\prime} 5^{\prime} H\right]$ flavonoid 3,5-hydroxylase; [F3'MO] flavonoid 3'-monooxygenase; [FS] flavanol synthase; $\left[F S F 3^{\prime} H\right]$ flavanol synthase flavanone 3-hydroxylase; $[L D O X]$ leucoanthocyanidin dioxygenase; [LAR] leucoanthocyanidin reductase; [MFMT] malonyl: flavonoid malonyltransferase. Gene number (if applicable) is shown before a hyphen; whereas numbers after a hyphen link transcripts with the same gene number (- $\underline{\underline{x}})$; while different splice variants (if applicable) of the same gene are separated by the number after the second hyphen (-x-x). For example, PPO-1-1 and PPO-1-2 indicate that there are two transcripts annotated as PPO (linked by 1 ), but they were classified as being a different isomer (indicated by 1 and 2 ).

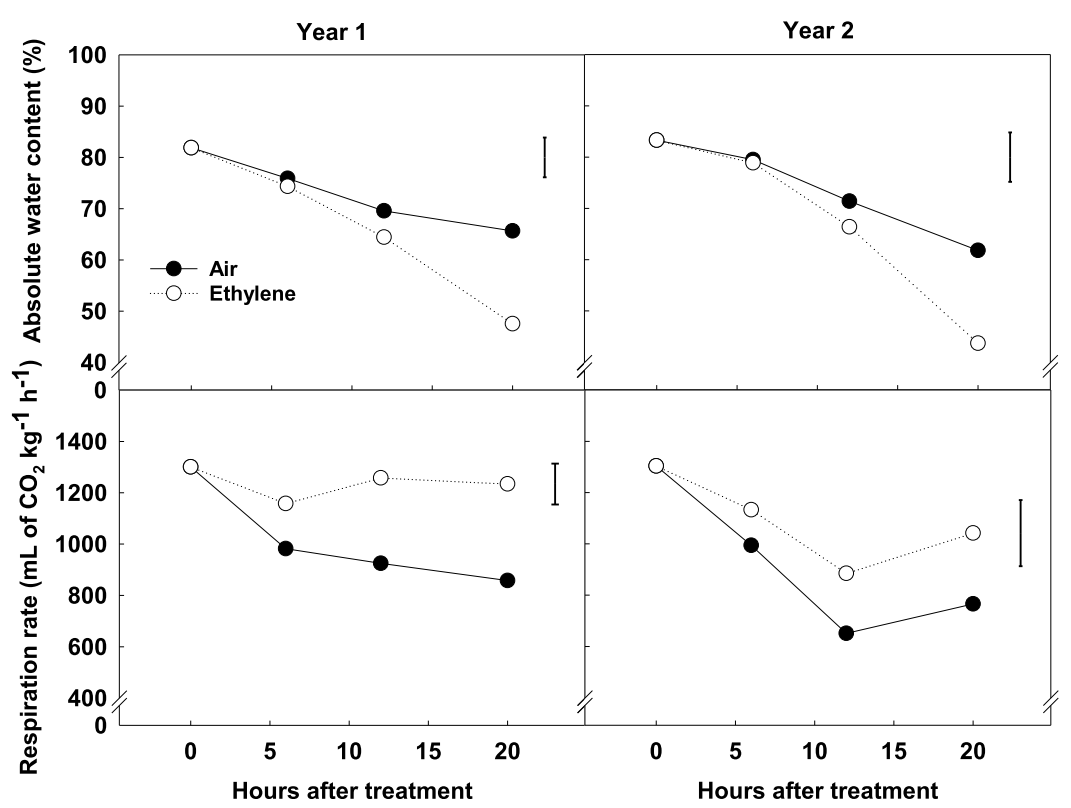

Figure 5. Physiological response of $3 \mathrm{LB}$ tea shoots during simulated withering conditions. Absolute water content and respiration rate as $\mathrm{CO}_{2}$ production of three leaves and a bud (3LB) tea samples (C. sinensis-Sri Lanka), cv. clone 2 subjected to $10 \mu \mathrm{L} \mathrm{L}^{-1}$ ethylene supplementation (ethylene) and air (control) during simulated withering. Least significant difference $\left(\mathrm{LSD}_{0.05}\right)$ bars for the interaction treatment $\times$ hours after treatment are shown. 
FLAVONOID BIOSYNTHESIS

\section{PHENYLPROPANOID BIOSYNTHESIS}
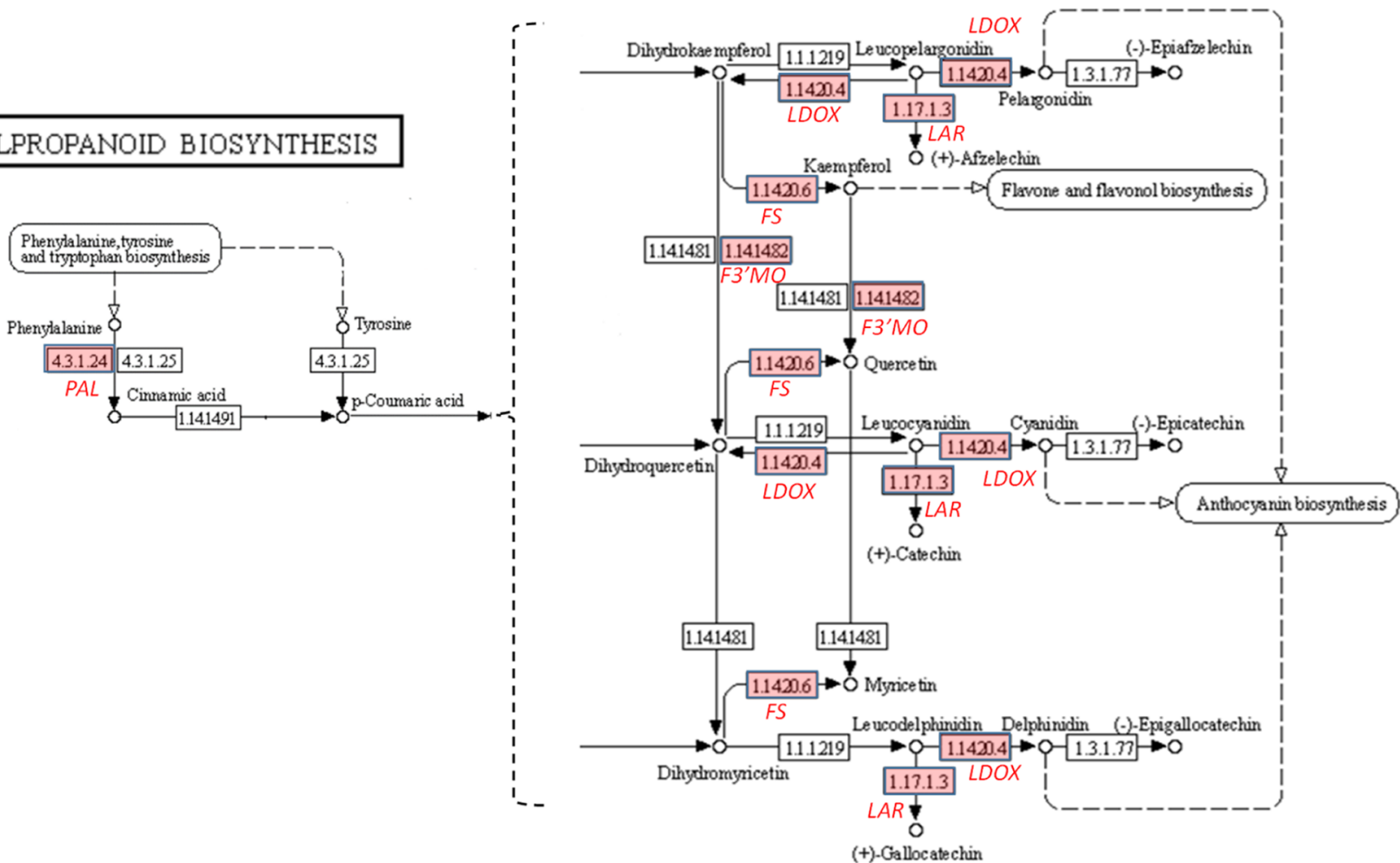

Figure 6. KEGG pathway showing key sections from phenylpropanoid and flavonoid biosynthesis. EC numbers highlighted in red represent transcripts upregulated by UV-C treatment in fresh tea shoots. [PAL] phenylalanine ammonia lyase; [F3'MO] flavonoid $3^{\prime}$-monooxygenase; [FS] flavanol synthase; $[L D O X]$ leucoanthocyanidin dioxygenase; $[L A R]$ leucoanthocyanidin reductase. Adapted from KEGG reference pathway.

withered samples), indicating that senescence during withering had been accelerated (Figure 5).

3.6. Differential Gene Expression Associated with Ethylene Treatment. A limited number of transcripts were DE following simulated withering $(6 \mathrm{~h})$ in air versus ethylene $\left(10 \mu \mathrm{L} \mathrm{L}^{-1}\right)$ (Supporting Information Data). As expected, a large proportion of these were related to abiotic stress responses, especially dehydration responses (viz. EXPANSINLIKE B1). There was also one gene associated with the ABA pathway; an $\mathrm{ABC}$ transporter $\mathrm{G}$ family member 25 ( $\left.A B C^{\prime} G 25\right)$ (Supporting Information Figure S5A) which showed ca. 3 log $\mathrm{FC}$ upregulation in ethylene relative to air and is known to have ABA-efflux activity. ${ }^{64} \mathrm{~A}$ higher number of DE transcripts were upregulated during the first $6 \mathrm{~h}$ of the withering, regardless of the treatment applied. Thus, four genes were annotated as abscisic acid $8^{\prime}$-hydroxylase $1\left(A B A 8^{\prime} H 1\right)$ and had the highest upregulation (ca. $8 \log$ FC) in air and ethylene after $6 \mathrm{~h}$ withering relative to baseline (before treatment application $[0 \mathrm{~h}]$ ) (Supporting Information Figure S5A).

Ethylene treatment also resulted in the upregulation (log FC of $c a .2$ ) of a number of transcripts within the ethylene signaling pathway of withered tea shoots: one transcript annotated as EIN4-like (ethylene receptor), four transcripts annotated as ethylene insensitive (EIN) 3-F box 1 (EIN3Fbox 1), and two annotated as ETHYLENE RESPONSE FACTORS ERF098-like-1 and ERF1B-like-2 (Supporting Information data). The majority of the 77 annotated transcripts associated with the ethylene pathway were upregulated during withering in both air and ethylene samples relative to baseline. That said, the three transcripts annotated as an ethylene-responsive transcription factor 098-like (ERF098-like) had greater upregulation in ethylene samples (ca. $7 \log$ FC) relative to the baseline, compared to air (ca. 5 $\log$ FC) (Supporting Information Figure S5B). Twelve transcripts were annotated as 1-aminocyclopropane-1-carboxylate oxidase $[A C O]$ and four annotated as 1-aminocyclopropane-1-carboxylate synthase $[A C S]$. Two isoforms of ACO4 were only upregulated relative to baseline in ethylene withered samples. ACS transcripts showed similar log FC in expression for both air and ethylene at $6 \mathrm{~h}$ relative to baseline.

Only one transcript annotated as INDOLE-3-ACETIC ACID-AUXIN-RESPONSIVE 29-like [IAA29-like-2] was upregulated in ethylene $6 \mathrm{~h}$ relative to air $6 \mathrm{~h}$; whilst a $W A T$ (WALLS ARE THIN-vacuolar auxin transport facilitator) transcript was downregulated (Supporting Information Figure S5C). Most of the transcripts annotated as belonging to the auxin pathway (a total of 101) were downregulated during simulated withering in both air and ethylene samples relative to baseline including auxin-induced 1 [AUX1] auxin-binding $[A B P]$ and auxin response factor $[A R F]$ transcripts (Supporting Information Figure S5C). Three IAA-amino acid hydrolase transcripts [ILR16-like], two IAA26-like transcripts, and an auxin binding transcript $[A B P 19 A-1]$ were upregulated (ca. 2$4 \log \mathrm{FC}$ ) by ethylene after $6 \mathrm{~h}$ withering relative to baseline, whereas no significant differential expression was observed for air $6 \mathrm{~h}$ relative to baseline. 


\section{DISCUSSION}

Tea withering is the first of the four steps needed for black tea production. Although a key stage, and despite the increasing demand for improved tea quality, withering methods have remained unchanged for many years. In this work, we evaluated the potential of either ethylene supplementation or UV-C irradiation as post-harvest techniques to enhance leaf senescence and improve catechin oxidation upon withering. A de novo transcriptome was assembled from 27 C. sinensis shoot samples in order to elucidate the mechanisms behind the physical and biochemical changes during simulated withering. Although the draft tea genome is available ${ }^{4}$ and there are recent transcriptomic studies where tea physiology under abiotic stresses has been investigated, ${ }^{44}$ we believe that our $d e$ novo analysis was required to generate an accurate transcriptome, which unambiguously represents the investigated $C$. sinensis var. assamica cv. Clone 2 line expression data under the different withering treatments. These data were integrated with the current tea genome information for downstream analyses.

4.1. UV-C Increased the Oxidation of Catechins into TFs in Whole Tea Leaves. Application of UV-C enhanced the withering process. However, in contrast to increasing moisture loss with ethylene supplementation, the mechanisms associated with the effects of UV-C were linked to an increase in oxidation processes.

Genes annotated as $P A L, P P O$, and $P O$, which are all plant defence-related enzymes, were $\mathrm{DE}$ in UV-C treated shoots during simulated withering. PAL is the initial enzyme in the phenylpropanoid pathway responsible for catalyzing the conversion of phenyl alanine into cinnamic acid. ${ }^{45}$ Upregulation of 11 transcripts annotated, as PAL as well as 14 flavonoid biosynthesis transcripts (viz. F3'MO, LDOX, LAR and FS), suggests that the flavonoid biosynthesis pathway was activated following UV-C exposure (Figure 6). This is in agreement with Lin et al. $(2021)^{46}$ who investigated the impact of ambient UV$\mathrm{B}$ on tea leaves. Their data indicated that the absence of ambient UV-B light downregulated the abundance of genes involved in the flavanol biosynthesis pathway, including CsPAL and CsLAR; whilst UV-C supplementation of tea cuttings resulted in flavanol, and cultivar-specific catechin accumulation. Although an increase in catechin content was not detected in the present study, this may have been masked by the rapid conversion of catechins into TF, which coincided with color changes from green to brown. Tea polyphenols are responsible for the coppery brown/black color characteristic of black tea, ${ }^{47}$ and significantly contribute toward the final black tea quality. ${ }^{48,49}$ It is likely that TFs were also being rapidly converted into larger tea polymers called TRs; however, these could not be measured because there is currently no accurate method to quantify TRs due to the complex mixture of heterogeneous polymers. ${ }^{48}$ That said, TR can be further condensed into larger polyphenols called theabrownins (FBs). TBs are typically produced during post-fermentation in $\mathrm{Pu}$-erh teas giving tea leaves an even darker colour. ${ }^{50} \mathrm{We}$ can then hypothesize that TB could be formed with high UV-C treatment doses and that they may be responsible for the darkening of the tea leaves observed in the experiment herein. During traditional tea processing, catechins are oxidized into larger tea polyphenols during the fermentation (oxidation) stage. However, the results herein indicate that UV-C treatment can initiate the oxidation stage during the withering process, which has the potential to speed up the tea manufacturing process. Moreover, and similarly to what was postulated by the application of UV-B light (longer wavelengths) on tea cuttings, ${ }^{46}$ the use of UV-C light during tea withering could be used to enhance the amount of flavor compounds in tea shoots, which may further impact on the quality of tea infusions.

Increased oxidation of catechins was also supported by transcriptomic analysis where $\mathrm{PO}$ and $\mathrm{PPO}$ transcripts were upregulated in UV-C-treated shoots. The main PO transcripts upregulated were from the subfamily of secreted class III peroxidases. These peroxidases are located in the cell wall, apoplast, and vacuole and have a diverse role throughout plant growth and development; including $\mathrm{H}_{2} \mathrm{O}_{2}$ generation and activation of cell death (peroxidase 2), and lignification (peroxidation 4). ${ }^{51-53}$ They are also efficient components of the antioxidant system which is induced in response to environmental stress such as high light intensity. ${ }^{53}$ In UV-C treated shoots, the peroxidase annotated as $P x 6$ had the highest logfold increase at both time points compared to control. It is plausible that this could be the main enzyme responsible for the oxidation of catechin to TF. However, the precise function and mechanism of action of this peroxidase still lacks clarification becauses no physiological or biochemical response has been reported in the literature. The two PPO transcripts upregulated in response to UV-C ${ }_{15}$ may also have contributed toward the formation of TF and TR. PPO genes are found to be differentially induced in response to wounding, pathogens or herbivores as well as to signaling molecules (e.g., ethylene and salicylic acid) indicating that these genes play a defence role. PPO activity is necessary for black tea fermentation where catechins are oxidized to $o$-diquinones. ${ }^{45}$

In addition to an increase in oxidation transcripts, cell decompartmentalization following membrane damage was also likely to have occurred. Evidence to support this was shown in year 2 where overall higher total conductivity readings (by $c a$. 1.5 -fold) were recorded for $\mathrm{UV}-\mathrm{C}_{15}$ leaves indicating greater electrolyte leakage. Ruptured membranes following exposure to UV-C would bring oxidative enzymes (PO and PPO) into contact with substrates (e.g., catechins) generating higher levels of oxidation products (e.g., TF and TR). Alterations in membrane permeability leading to oxidation was suggested to explain the color changes in spinach leaves that developed severe browning following UV-C treated samples (7.94 and $11.35 \mathrm{~kJ} \mathrm{~m}^{-2} \mathrm{UV}-\mathrm{C}$ ) after 10 days at 5 and $8{ }^{\circ} \mathrm{C} .{ }^{27} \mathrm{~A}$ similar color change occurred in tea shoots exposed to UV-C, where leaves changed from green to red or brown, indicative of oxidation. Low doses of UV-C are likely to have caused controlled apoptosis; while high UV-C caused uncontrolled chaotic necrosis resulting in the plasma membrane losing its ability to osmoregulate, such that water enters the cells and the cell bursts. ${ }^{54}$ This could explain the increase in conductivity in high UV-C $\left(15 \mathrm{~kJ} \mathrm{~m}^{-2}\right)$ treated tea shoots.

Differential expression of transcripts associated with cell membrane channels/proteins was also observed in UV-C treated shoots compared to control, which could have contributed to an increase in conductivity through ion flux and apoptosis. After $12 \mathrm{~h}$ withering, expression of MS 10 ion channels genes were upregulated in UV-C. These are transmembrane proteins that directly couple mechanical stimuli to ion flux that can perceive various mechanical stimuli such as osmotic pressure, touch, gravity, and pathogenic invasion. ${ }^{55}$ It is likely these proteins reduced osmotic stress in UV-C leaves. Furthermore, a significant upregulation of 
CNGC1 occurred in UV-C leaves with expression continuing to increase with time reaching an 8-log fold increase at $12 \mathrm{~h}$ compared to control. CNGC channels play an adaptive role in plants to restore cellular homeostasis promoting survival. ${ }^{56}$ Activation of $C N G C 1$ is involved in the uptake of $\mathrm{Ca}^{2+}$, which is a secondary messenger generated in response to a stress stimulus that can trigger a signal transduction cascade. ${ }^{56}$ However, a prolonged increase and high level of $\mathrm{Ca}^{2+}$ has been linked to apoptosis. ${ }^{57}$ Furthermore, another study has shown exposure to UV-B light increased $\mathrm{Ca}^{2+}$ levels in parsley cells followed by subsequent changes in gene expression. ${ }^{58}$ Consequently, these changes in the cell membranes of UV-C treated shoots may have been another contributing factor responsible for the observed rise in conductivity.

The initiation of oxidation (fermentation) during typical tea processing requires cells to be ruptured (by cutting/rolling) to allow de-compartmentalization; however, this oxidation process appears to have occurred in UV-C treated-leaves (whilst the leaves remained whole), allowing for tea shoots to remain intact and whole during tea processing. Dried tea leaves that remain intact or partially broken are regarded as being a higher quality tea. ${ }^{59}$ Consequently, the use of UV-C to initiate oxidation and enzyme decompartmentalization during withering whilst the tea leaf remains whole could be used to shorten the 'fermentation' process during tea manufacture. Furthermore, tea quality is also known to be influenced by plucking standards (viz. $2 \mathrm{LB}$ or $3 \mathrm{LB}){ }^{13}$ which was shown in this work, where significantly lower catechin concentrations were observed in the large leaf compared to the bud, similar to that previously reported in Collings et al. (2019). ${ }^{3}$ Therefore, the treatment mentioned in Supporting Information of UV-C during withering could provide tea growers with the potential to use lower grade shoots for tea production to enhance oxidation thus producing high enough TF and TR levels. This also has the potential to reduce waste and improve marketable yields.

4.2. Exogenous Ethylene Accelerates Tea Withering Rate. Ethylene supplementation at $10 \mu \mathrm{L} \mathrm{L}^{-1}$ for $20 \mathrm{~h}$ during withering at $25{ }^{\circ} \mathrm{C}$, significantly increased the metabolic rate of tea shoots compared to air. Thus, there were a number of DE genes related to abiotic stress responses. For example, EXPANSIN-LIKE B1 has been shown to elicit a response in drought stress in Brassica rapa ${ }^{60}$ and potato. ${ }^{61}$ Coinciding with the increase in metabolic rate, ethylene treated leaves also experienced greater moisture loss, which could be an inhibitory effect of ethylene on ABA-induced stomatal closure resulting in increased transpiration. ${ }^{62}$ As demonstrated by Tanaka et al. (2005), ${ }^{63}$ when gaseous ethylene or the ethylene precursor 1aminocyclopropane-1-carboxylic acid (ACC) was applied in combination with ABA to isolated epidermal peels in Arabidopsis, the stomata remained open. Our transcriptomic analysis showed that $A B C^{\prime} G 25$ was upregulated in ethylenetreated tea shoots. $A B C^{\prime} G 25$ is a known $A B A$ transporter ${ }^{64}$ which transfers $\mathrm{ABA}$ from the cytoplast to the apoplast, ${ }^{64}$ therefore decreasing cellular ABA levels. This may further result in a reduced sensitivity to $\mathrm{ABA}$-induced responses. However, Kuromori et al. $(2010)^{65}$ demonstrated that AtABCG25-overexpressing plants had slower leaf water loss compared to wild-type plants. Therefore, we cannot confirm that the physiological changes observed in ethylene-treated tea shoots are mediated by changes in the $\mathrm{ABA}$ signaling pathway via $A B C^{\prime} G 25$.
Concomitantly to the impact on the ABA pathway, the upregulation of the ethylene receptor EIN4 and two ethylene transcription factors (EIN3-Fbox and ERF1B) in ethylenetreated samples may have enhanced the ethylene response of tea shoots, such as the inhibition of stomata closure, and leaf senescence. Related to the latter, and in addition to moisture loss, color changes also occurred in ethylene-treated tea shoots; where leaves gradually changed from an intense green color to dull green/slightly yellow. This loss of a green color is associated with chloroplast degradation and is an integral part of leaf senescence. ${ }^{66}$

The current research, therefore, provides a de novo transcriptome for tea shoots during simulated withering. It was established that UV-C $\left(15 \mathrm{~kJ} \mathrm{~m}^{-2}\right)$ treatment applied to fresh tea leaves (C. sinensis var. assamica $\mathrm{cv}$. Clone 2) promoted rapid changes in color from green to red/brown coinciding with enhanced oxidation of catechins into larger polyphenols (viz. TFs); this could be explained by the upregulation of peroxidases (viz. Px2, $P \times 4$ and $P \times 6$ ) and polyphenol oxidases (PPO-1) found in UV-C treated tea shoots. The decompartmentalization that would result from cellular damage induced by UV-C irradiation was evidenced by enhanced conductivity. Ethylene supplementation of detached green tea shoots accelerated withering by increasing respiration rate and water loss. At the transcriptomic level, ethylene supplementation affected $A B A$, ethylene, and auxin signaling pathways by upregulating the $A B A$ transporter $A B C-G 25$, the ethylene receptor EIN4-like and the transcriptor factor EIN3-Fbox-like, and downregulating the vacuolar auxin transport facilitator WAT. Ethylene and UV-C treatments could potentially shorten withering time while enhancing the accumulation of flavor compounds, providing a higher quality tea with a reduced processing time.

\section{ASSOCIATED CONTENT}

\section{SI Supporting Information}

The Supporting Information is available free of charge at https://pubs.acs.org/doi/10.1021/acs.jafc.1c02876.

Assembly metrics for the de novo assembly of $C$. sinensis withering transcriptome, number of transcripts that are up- or down-regulated as a result of the ethylene or UV$\mathrm{C}$ treatment, individual pathway ratios from the metabolic pathway enrichment analysis for ethylene treatment (ethylene_6h) vs control (control_Air_6h), individual pathway ratios from the metabolic pathway enrichment analysis for UV-C treatment at $4 \mathrm{~h}$ (UVC_4h) vs control at $4 \mathrm{~h}$ (control_UV-C_4h), individual pathway ratios from the metabolic pathway enrichment analysis for UV-C treatment at $12 \mathrm{~h}$ (UV-C 12h) vs control at $12 \mathrm{~h}$ (UV-C_4h), unsupervised transcriptomic exploratory analysis of $C$. sinensis, functional annotation of C. sinensis withering transcriptome, gene ontology classification for $C$. sinensis withering transcriptome,catechins concentration and distribution in C. sinensis $2 \mathrm{LB}$ shoots. DE transcripts of $C$. sinensis $3 \mathrm{Lb}$ shoots during simulated ethylene withering (PDF)

\section{AUTHOR INFORMATION}

\section{Corresponding Author}

Leon A. Terry - Plant Science Laboratory, Cranfield University, Cranfield, Bedfordshire MK43 OAL, U.K.; 
다이.org/0000-0001-7871-5514; Phone: 00441234

750111; Email: 1.a.terry@cranfield.ac.uk

\section{Authors}

Emma R. Collings - Plant Science Laboratory, Cranfield University, Cranfield, Bedfordshire MK43 OAL, U.K.

M. Carmen Alamar - Plant Science Laboratory, Cranfield University, Cranfield, Bedfordshire MK43 OAL, U.K.; (1) orcid.org/0000-0001-9235-9288

Maria Bogaerts Márquez - Bioinformatics Group, Cranfield University, Cranfield, Bedfordshire MK43 OAL, U.K.

Sofia Kourmpetli - Plant Science Laboratory, Cranfield University, Cranfield, Bedfordshire MK43 OAL, U.K.

Zoltan Kevei - Plant Science Laboratory, Cranfield University, Cranfield, Bedfordshire MK43 OAL, U.K.

Andrew J. Thompson - Plant Science Laboratory, Cranfield University, Cranfield, Bedfordshire MK43 OAL, U.K.

Fady Mohareb - Bioinformatics Group, Cranfield University, Cranfield, Bedfordshire MK43 OAL, U.K.

Complete contact information is available at:

https://pubs.acs.org/10.1021/acs.jafc.1c02876

\section{Author Contributions}

E.R.C and M.C.A. are shared main co-authors and have equally contributed to the manuscript; they designed and performed the experimental work, analyzed the data and wrote the article with contributions from all authors. M.B.M. contributed to the RNA-seq analysis. S.K. contributed to the interpretation of the results and the reviewing of the manuscript; Z.K. contributed to the RNA extraction and the reviewing of the manuscript. F.M. carried out the RNA-seq analysis. L.A.T., (F.M. and A.J.T) conceived the research plans and supervised the work. L.A.T. agrees to serve as the author responsible for contact.

\section{Notes}

The authors declare no competing financial interest.

The underlying physiology and biochemical data can be accessed at https://doi.org/10.17862/cranfield.rd.14038814. RNA-Seq repository is on NCBI-SRA (Bioproject: PRJNA705055, BioSample: SAMN18061975).

\section{ACKNOWLEDGMENTS}

The authors would like to thank the Biotechnology and Biological Sciences Research Council (BBSRC; BB/J500665/ 1) and Unilever R\&D, UK, for funding this research. Special thanks are given to Dr Katherine Cools for her contribution during experimental delivery; and Dr Pat Bellamy for providing statistical support.

\section{REFERENCES}

(1) Preedy, V. Tea in Health and Disease Prevention; Elsevier: London, 2012.

(2) Deb, S.; Jolvis Pou, K. R. A Review of withering in the processing of black tea. J. Biosyst. Eng. 2016, 41, 365-372.

(3) Collings, E. R.; Alamar, M. C.; Redfern, S.; Cools, K.; Terry, L. A. Spatial changes in leaf biochemical profile of two tea cultivars following cold storage under two different vapour pressure deficit (VPD) conditions. Food Chem. 2019, 277, 179-185.

(4) Xia, E.; et al. The tea plant reference genome and improved gene annotation using long-read and paired-end sequencing data. Sci. Data 2019, 6, 122.

(5) Li, H.; Liu, Z.-W.; Wu, Z.-J.; Wang, Y.-X.; Teng, R.-M.; Zhuang, J. Differentially expressed protein and gene analysis revealed the effects of temperature on changes in ascorbic acid metabolism in harvested tea leaves. Hortic. Res. 2018, 5, 65.
(6) Jiang, C.-K.; Ma, J.-Q.; Liu, Y.-F.; Chen, J.-D.; Ni, D.-J.; Chen, L. Identification and distribution of a single nucleotide polymorphism responsible for the catechin content in tea plants. Hortic. Res. 2020, 7, 24.

(7) Wang, Y.; et al. Genome-wide identification, characterisation and expression analysis of nucleotide-binding leucine-rich repeats gene family under environmental stresses in tea (Camellia sinensis). Genomics 2020, 112, 1351-1362.

(8) Xia, E.; et al. Resequencing of 81 diverse accessions provide insights into its genome evolution and adaptation. Mol. Plant 2020, 13, $1013-1026$.

(9) Wang, X. C.; et al. Global transcriptome profiles of Camellia sinensis during cold acclimation. BMC Genom. 2013, 14, 415.

(10) Li, C.-F.; et al. Global transcriptome and gene regulation network for secondary metabolite biosynthesis of tea plant (Camellia sinensis). BMC Genom. 2015, 16, 560.

(11) Zhang, H.-B.; Xia, E.-H.; Huang, H.; Jiang, J.-J.; Liu, B.-Y.; Gao, L.-Z. De novo transcriptome assembly of the wild relative of tea tree (Camellia taliensis) and comparative analysis with tea transcriptome identified putative genes associated with tea quality and stress response. BMC Genom. 2015, 16, 298.

(12) Wu, Z.-J.; Ma, H.-Y.; Zhuang, J. iTRAQ-based proteomics monitors the withering dynamics in postharvest leaves of tea plant (Camellia sinensis). Mol. Genet. Genomics 2018, 293, 45-59.

(13) Okinda Owuor, P.; Obanda, M. The changes in black tea quality due to variations of plucking standard and fermentation time. Food Chem. 1998, 61, 435-441.

(14) Kim, J. H.; Chung, K. M.; Woo, H. R. Three positive regulators of leaf senescence in Arabidopsis, ORE1, ORE3 and ORE9, play roles in crosstalk among multiple hormone-mediated senescence pathways. Genes Genomics 2011, 33, 373-381.

(15) Grbic, V.; Bleecker, A. B. Ethylene regulates the timing of leaf senescence in Arabidopsis. Plant J. 1995, 8, 595-602.

(16) López-Gálvez, G.; Saltveit, M.; Cantwell, M. Wound-induced phenylalanine ammonia lyase activity: factors affecting its induction and correlation with the quality of minimally processed lettuces. Postharvest Biol. Technol. 1996, 9, 223-233.

(17) Borochov, A.; Spiegelstein, H.; Philosoph-Hadas, S. Ethylene and flower petal senescence: Interrelationship with membrane lipid catabolism. Physiol. Plantarum 1997, 100, 606-612.

(18) Rinaldi, R.; Amodio, M. L.; Colelli, G. Effect of temperature and exogenous ethylene on the phyiological and quality traits of purslane (Portulaca olearacea L.) leaves during storage. Postharvest Biol. Technol. 2010, 58, 147-156.

(19) Jia, Y.; Li, W. Characterisation of lipid changes in ethylenepromoted senescence and its retardation by suppression of phospholipase D $\delta$ in Arabidopsis leaves. Front. Plant Sci. 2015, 6, 1045.

(20) Hartman, S.; Sasidharan, R.; Voesenek, L. A. C. J. The role of ethylene in metabolic acclimations to low oxygen. New Phytol. 2021, 229, 64-70.

(21) Zhang, W.; Jiang, W. UV treatment improved the quality of postharvest fruits and vegetables by inducing resistance. Trends Food Sci. Technol. 2019, 92, 71-80.

(22) Terry, L.; Joyce, D. C. Elicitors of induced disease resistance in postharvest horticultural crops: a brief review. Postharvest Biol. Technol. 2004, 32, 1-13.

(23) Crupi, P.; Pichierri, A.; Basile, T.; Antonacci, D. Postharvest stilbenes and flavonoids enrichment of table grapecv Redglobe (Vitis vinifera L.) as affected by interactive UV-C exposureand storage conditions. Food Chem. 2013, 141, 802-808.

(24) Collings, E. R.; Alamar Gavidia, M. C.; Cools, K.; Redfern, S.; Terry, L. A. Effect of UV-C on the physiology and biochemical profile of fresh Piper nigrum berries. Postharvest Biol. Technol. 2018, 136, $161-165$.

(25) Adetuyi, F. O.; Karigidi, K. O.; Akintimehin, E. S. Effect of postharvest UV-C treatments on the bioactive components, antioxidant and inhibitory properties of clerodendrum volubile leaves. J. Saudi Soc. Agric. Sci. 2020, 19, 7-13. 
(26) Allende, A.; McEvoy, J. L.; Luo, Y.; Artés, F.; Wang, C. Y. Effectiveness of two-sided UV-C treatments in inhibiting natural microflora and extending the shelf-life of minimally processed 'Red Oak Leaf lettuce. Food Microbiol. 2006, 23, 241-249.

(27) Artés-Hernández, F.; Escalona, V. H.; Robles, P. A.; MartínezHernández, G. B.; Artés, F. Effect of UV-C radiation on quality of minimally processed spinach leaves. J. Sci. Food Agric. 2009, 89, 414421.

(28) Alamar, M. C.; Collings, E.; Cools, K.; Terry, L. A. Impact of controlled atmosphere scheduling on strawberry and imported avocado fruit. Postharvest Biol. Technol. 2017, 134, 76-86.

(29) Griffin, D. H. Fungal Physiology, 2nd ed.; Wiley: New York, 1994.

(30) Andrews, S. FastQC: a quality control tool for high throughput sequence data. 2010, Available online at: http://www.bioinformatics. babraham.ac.uk/projects/fastqc (accessed: December 2019).

(31) Grabherr, M. G.; et al. Full-length transcriptome assembly from RNA-Seq data without a reference genome. Nat. Biotechnol. 2011, 29, 644-652.

(32) Haas, B. J.; et al. De novo transcript sequence reconstruction from RNA-seq using the Trinity platform for reference generation and analysis. Nat. Protoc. 2013, 8, 1494-1512.

(33) Li, W.; Godzik, A. Cd-hit: a fast program for clustering and comparing large sets of protein or nucleotide sequences. Bioinformatics 2006, 22, 1658-1659.

(34) Dobin, A.; et al. STAR: ultrafast universal RNA-seq aligner. Bioinformatics 2013, 29, 15-21.

(35) Conesa, A.; Götz, S. Blast2GO: A comprehensive suite for functional analysis in plant genomics. Int. J. Plant Genom. 2008, 2008, 619832.

(36) Jones, P.; et al. InterProScan 5: genome-scale protein function classification. Bioinformatics 2014, 30, 1236-1240.

(37) Li, B.; Dewey, C. N. RSEM: accurate transcript quantification from RNA-Seq data with or without a reference genome. BMC Bioinf. 2011, 12, 323.

(38) Law, C. W.; Chen, Y.; Shi, W.; Smyth, G. K. voom: Precision weights unlock linear model analysis tools for RNA-seq read counts. Genome Biol. 2014, 15, R29.

(39) Xia, E.-H.; et al. The tea tree genome provides insights into tea flavor and independent evolution of caffeine biosynthesis. Mol. Plant 2017a, 10, 866-877.

(40) Xia, E.-H.; et al. The Tea Tree Genome Database, 2017.

(41) Langmead, B.; Salzberg, S. L. Fast gapped-read alignment with Bowtie 2. Nat. Methods 2012, 9, 357-359.

(42) Trapnell, C.; Pachter, L.; Salzberg, S. L. TopHat: discovering splice junctions with RNA-Seq. Bioinformatics 2009, 25, 1105-1111.

(43) Trapnell, C.; et al. Transcript assembly and quantification by RNA-Seq reveals unannotated transcripts and isoform switching during cell differentiation. Nat. Biotechnol. 2010, 28, 511-515.

(44) Xia, E.-H.; Tong, W.; Wu, Q.; Wei, S.; Zhao, J.; Zhang, Z.-Z.; Wei, C.-L.; Wan, X.-C. Tea plant genomics: achievements, challenges and perspectives. Hortic. Res. 2020, 7, 7.

(45) Samynathan, R.; Perisamy, S. K.; Sudhakar, G.; Anitha, J.; Geetha, S.; Mohankumar, P.; Gopalakrishnan, V. K. Biochemical and molecular analysis of Camellia sinensis (L.) O. Kuntze tea from the selected P/11/15 clone. J. Genet. Eng. Biotechnol. 2016, 14, 69-75.

(46) Lin, N.; Liu, X.; Zhu, W.; Cheng, X.; Wang, X.; Wan, X.; Liu, L. Ambient ultraviolet $\mathrm{B}$ signal modulates tea flavor characteristics via shifting a metabolic flux in flavonoid biosynthesis. J. Agric. Food Chem. 2021, 69, 3401-3414.

(47) Bhattacharyya, N.; et al. Monitoring of black tea fermentation process using electronic nose. J. Food Eng. 2007, 80, 1146-1156.

(48) Kuhnert, N.; Drynan, J. W.; Obuchowicz, J.; Clifford, M. N.; Witt, M. Mass spectrometric characterization of black tea thearubigins leading to an oxidative cascade hypothesis for thearubigin formation. Rapid Commun. Mass Spectrom. 2010, 24, 3387-3404.

(49) Sabhapondit, S.; Karak, T.; Bhuyan, L. P.; Goswami, B. C.; Hazarika, M. Diversity of catechin in Northeast Indian tea cultivars. Sci. World J. 2012, 2012, 485193.
(50) Jiang, Y.; Hua, J.; Wang, B.; Yuan, H.; Ma, H. Effects of variety, season and region on theaflavin content of fermented Chinese Congou black tea. J. Food Qual. 2018, 2018, 5427302.

(51) Cosio, C.; Dunand, C. Specific functions of individual class III peroxidase genes. J. Exp. Bot. 2009, 60, 391-408.

(52) Shigeto, J.; Tsutsumi, Y. Diverse functions and reactions of class III peroxidases. New Phytol. 2016, 209, 1395-1402.

(53) Jovanovic, S. V.; Kukavica, B.; Vidović, M.; Morina, F.; Menckhoff, L. Antioxidants and Antioxidant Enzymes in Higher Plants; Gupta, D., Palma, J., Corpas, F., Eds.; Springer: Cham, 2018.

(54) Reape, T. J.; Molony, E. M.; McCabe, P. F. Programmed cell death in plants: distinguishing between different modes. J. Exp. Bot. 2008, 59, 435-444.

(55) Basu, D.; Haswell, E. S. Plant mechanosensitive ion channels: an ocean of possibilities. Curr. Opin. Plant Biol. 2017, 40, 43-48.

(56) Jha, S. K.; Sharma, M.; Pandey, G. K. Role of cyclic nucleotide gated channels in stress management in plants. Curr. Genomics 2016, 17, 315-329.

(57) White, P. J.; Broadley, M. R. Calcium in plants. Ann. Bot. 2003, 92, 487-511.

(58) Frohnmeyer, H.; Loyall, L.; Blatt, M. R.; Grabov, A. Millisecond UV-B irradiation evokes prolonged elevation of cytosolic-free $\mathrm{Ca}^{2+}$ and stimulates gene expression in transgenic parsley cell cultures. Plant J. 1999, 20, 109-117.

(59) Tea: Bioactivity and Therapeutic Potential (Medicinal and Aromatic Plants-Industrial Profiles); Zhen, Y.-S., Ed.; CRC Press: Boca Raton, Florida: USA, 2002.

(60) Krishnamurthy, P.; Muthusamy, M.; Kim, J. A.; Jeong, M.-J.; Lee, S. I. Brassica rapa espansin-like B1 gene (BrEXLB1) regulate growth and development in transgenic Arabidopsis and elicits response to abiotic stresses. J. Plant Biochem. Biotechnol. 2019, 28, 437-446.

(61) Moon, K. B.; et al. Transcriptome profiling and characterisation of drought-tolerant potato plant (Solanum tuberosum L.). Mol. Cell. 2018, 41, 979-992.

(62) Daszkowska-Golec, A.; Szarejko, I. Open or close the gate stomata action under the control of phytohormones in drought stress conditions. Front. Plant Sci. 2013, 4, 138.

(63) Tanaka, Y.; Sano, T.; Tamaoki, M.; Nakajima, N.; Kondo, N.; Hasezawa, S. Ethylene inhibits abscisic acid-induced stomatal closure in Arabiposis. Plant Physiol. 2005, 138, 2337-2343.

(64) Park, Y.; et al. Spatial regulation of ABCG25, an ABA exporter, is an important component of the mechanism controlling cellular ABA levels. Plant Cell 2016, 28, 2528-2544.

(65) Kuromori, T.; et al. ABC transporter AtABCG25 is involved in abscisic acid transport and responses. Proc. Natl. Acad. Sci. U.S.A. 2010, 107, 2361-2366.

(66) Iqbal, N.; 'Khan, N. A.; Ferrante, A.; Trivellini, A.; Francini, A.; Khan, M. I. R. Ethylene role in plant growth, development and senescence: interaction with other phytohormones. Front. Plant Sci. $2017,8,475$. 